\title{
AI Based 3D Image Analysis with GeoDict
}

\author{
Andreas Griesser and Mathias Fingerle
}

Math2Market GmbH, Kaiserslautern, Rheinland-Pfalz, Germany

\section{Introduction}

Image analysis is on the verge of unleashing its full potential employing artificial intelligence (AI) for processing large datasets with a high information-depth. But the more complex the usage-scenarios become, the more specific are the challenges for AI-apporaches. Neural Networks (NN) must be trained for specific tasks, e.g. identifying a specific type of object. In this work, we present our concept to train NNs for such specific object identification and segmentation routines, giving two state-of-the-art examples: Firstly, we demonstrate the segmentation of a battery nickel-mangenese-cobalt (NMC) Cathode. Although they have the same grey value, a trained NN can separate the binder phase from the NMC grains and the particle statistics of the NMC can be further analyzed. Secondly, a NN trained for fiber identification can identify single glass fibers in a glass fiber re-enforced composite, giving information on length, diameter, contact points and many more. After image analysis, the geometry of the respective microstructure is characterized: We demonstrate porosimetry, granulometry and more on an industrial foam structure, which has relevance for e.g. bones as well. Above that, we simulate the complex mechanics of a large deformation on the foam. All this is performed with GeoDict - The Digital Material Laboratory.

\section{Method}

Segmenting certain feature in $\mu \mathrm{CT}$ scans can in many cases be challenging. To overcome this issue, we apply a machine learning base approached that can correctly even segment even for the human eye visually challenging areas by considering the full 3D neighborhood of an area. As with any machine learning base approach availability and quality of training data determines the quality of the results.

Using GeoDict we can generate a wast amount of training data containg all the information reaquired to even detect typically hard to segment and analyze in $\mu \mathrm{CT}$ scans. To do this we deplay various microstructure generators that are capable of generating e.g. fibrous, granular and foam like structures. After training the NN on these generated structures we us it on a real $\mu \mathrm{CT}$ scan. Using this method, we are able to segment phases that have no contrast in $\mu \mathrm{CT}$ e.g. Binder and Particles in the NMC cathode, and even label individual fibers in GFRP.

Using this, statistical properties such as the distributions of fiber length, fiber diameters, grain diameters, orientations, and other properties can easily be analyzed.

\section{Results}

In Figure 1, the image analysis of a battery NMC cathode [1] is shown. The cathode consists of active material, which stores the lithium, in this case NMC grains, and a binder phase, which usually consists of a plastic polymer and conductive additives. The two phases have the same grey value in the micoCT scan. A NN has been trained on digitally generated datasets to distinguish between binder and active material. A watershed algorithm then identifies each single grain and fits the grain with an elliptical shape. The statistics of the fitted grains like diameters, orientation, sphericity, and many more, can then be analyzed and used for modelling approaches. Creating a Statistical Digital Twin with powerful structure generators enables the development of new prototype battery materials. 
In Figure 2 the fiber analysis of a GFRP is shown. The infividual fibers a labeled using the NN and visualized in diffent colors in the left side. This reperesention allows to analyze all fiber properties such as diameter and length distributions easily. On The right the fiber diameter distribution is shown as an example.

\section{Conclusion}

We were successfully able to apply neural network trained on generated data to real $\mu \mathrm{CT}$ scans in multiple applications. The same process can be applied to individual cases not covered right now. GeoDict is offering the complete workflow from image processing over geometrical analysis up to complex numerical calculations for micro scale applications.
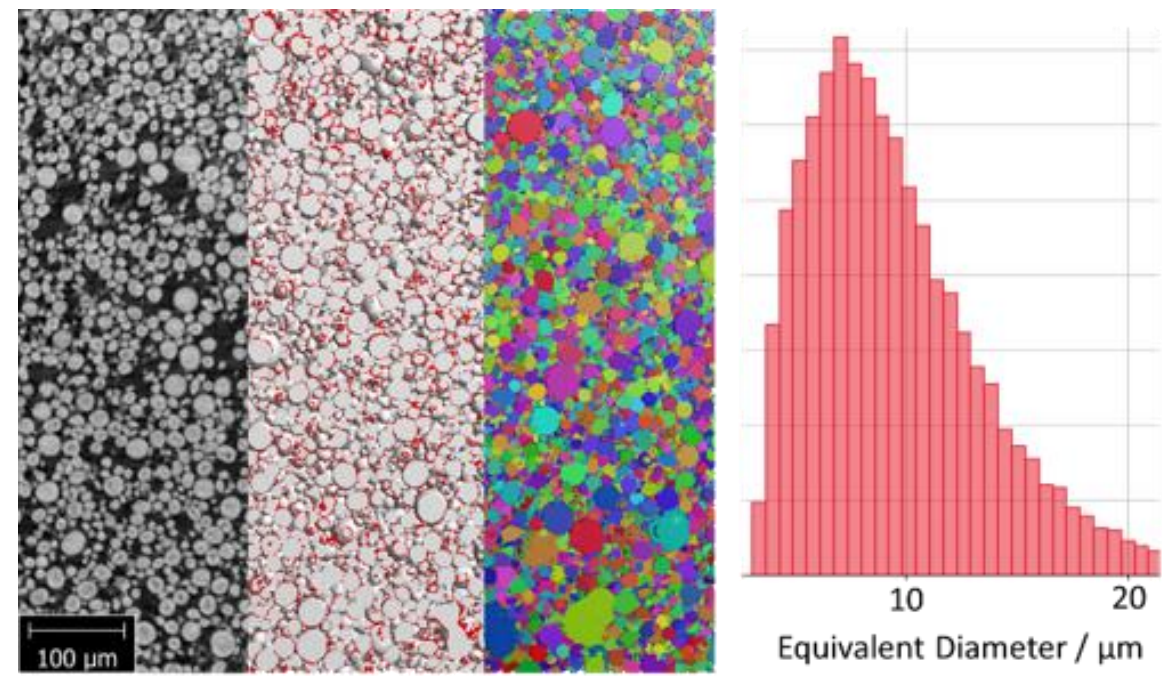

Equivalent Diameter / $\mu \mathrm{m}$

Figure 1. Visualization of the microCT Scan of a battery NMC cathode: Starting from the grey value image the binder is separated from the NMC grains and each grain can be analyzed individually, e.g. with respect to the diameter of the equivalent sphere.


Figure 2. Visualization of identified individual fibers in the microCT scan of a glass-fiber re-enforced composite and fiber statistics of the inner fiber diameter.

\section{References}

1. Ebner, Martin, et al. "Tortuosity anisotropy in lithium-ion battery electrodes." Advanced Energy Materials 4.5 (2014): 1301278. 